\title{
Characterization of Optimal Key Set Protocols (Extended Abstract)
}

\author{
Takaaki Mizuki $^{1}$, Hiroki Shizuya ${ }^{2}$, and Takao Nishizeki ${ }^{1}$ \\ 1 Graduate School of Information Sciences, Tohoku University, \\ Aoba-yama 05, Aoba-ku, Sendai 980-8579, Japan \\ \{mizuki,nishi\}@ecei.tohoku.ac.jp \\ 2 Education Center for Information Processing, Tohoku University, \\ Kawauchi, Aoba-ku, Sendai 980-8576, Japan \\ shizuya@ecip.tohoku.ac.jp
}

\begin{abstract}
Using a random deal of cards to players and a computationally unlimited eavesdropper, all players wish to share a common one-bit secret key which is information-theoretically secure from the eavesdropper. This can be done by the so-called key set protocol. In this paper we give a necessary and sufficient condition for a key set protocol to be "optimal," that is, to succeed always in sharing a one-bit secret key.
\end{abstract}

\section{Introduction}

Suppose that there are $k(\geq 2)$ players $P_{1}, P_{2}, \cdots, P_{k}$ and a passive eavesdropper, Eve, whose computational power is unlimited. All players wish to share a common one-bit secret key that is information-theoretically secure from Eve. Let $C$ be a set of $d$ distinct cards which are numbered from 1 to $d$. All cards in $C$ are randomly dealt to players $P_{1}, P_{2}, \cdots, P_{k}$ and Eve. We call a set of cards dealt to a player or Eve a hand. Let $C_{i} \subseteq C$ be $P_{i}$ 's hand, and let $C_{e} \subseteq C$ be Eve's hand. We denote this deal by $\mathcal{C}=\left(C_{1}, C_{2}, \cdots, C_{k} ; C_{e}\right)$. Clearly $\left\{C_{1}, C_{2}, \cdots, C_{k}, C_{e}\right\}$ is a partition of set $C$. We write $c_{i}=\left|C_{i}\right|$ for each $1 \leq i \leq k$ and $c_{e}=\left|C_{e}\right|$, where $|A|$ denotes the cardinality of a set $A$. Note that $c_{1}, c_{2}, \cdots, c_{k}$ and $c_{e}$ are the sizes of hands held by $P_{1}, P_{2}, \cdots, P_{k}$ and Eve respectively, and that $d=\sum_{i=1}^{k} c_{i}+c_{e}$. We call $\gamma=\left(c_{1}, c_{2}, \cdots, c_{k} ; c_{e}\right)$ the signature of deal $\mathcal{C}$. In this paper we assume that $c_{1} \geq c_{2} \geq \cdots \geq c_{k}$; if necessary, we rename the players. The set $C$ and the signature $\gamma$ are public to all the players and even to Eve, but the cards in the hand of a player or Eve are private to herself, as in the case of usual card games. This paper addresses protocols which make all the players share a common one-bit secret key information-theoretically securely using such a random deal of cards [23445610]. A reasonable situation in which such protocols are practically required is discussed in [4]6], and also the reason why we deal cards even to Eve is found there.

We consider a graph called a key exchange graph, in which each vertex $i$ represents a player $P_{i}$ and each edge $(i, j)$ joining vertices $i$ and $j$ represents a pair of players $P_{i}$ and $P_{j}$ sharing a one-bit secret key $r_{i j} \in\{0,1\}$. Refer to 
[8] for the graph-theoretic terminology. A connected graph having no cycle is called a tree. If the key exchange graph is a tree, then all the players can share a common one-bit secret key $r \in\{0,1\}$ as follows: an arbitrary player chooses a one-bit secret key $r \in\{0,1\}$, and sends it to the rest of the players along the tree; when player $P_{i}$ sends $r$ to player $P_{j}$ along an edge $(i, j)$ of the tree, $P_{i}$ computes the exclusive-or $r \oplus r_{i j}$ of $r$ and $r_{i j}$ and sends it to $P_{j}$, and $P_{j}$ obtains $r$ by computing $\left(r \oplus r_{i j}\right) \oplus r_{i j}$. For $k=2$, Fischer, Paterson and Rackoff give a protocol to form a tree, i.e. a graph having exactly one edge, as the key exchange graph by using a random deal of cards [2. Fischer and Wright extend this protocol for any $k \geq 2$, and formalize a class of protocols called "key set protocols," a formal definition of which will be given in the succeeding section 3 , 6. We say that a "key set protocol" works for a signature $\gamma$ if the protocol always forms a tree as the key exchange graph for any deal $\mathcal{C}$ having the signature $\gamma$.

Let $\Gamma_{k}$ be the set of all signatures of deals for $k$ players, where the total number $d$ of dealt cards is not fixed but takes any value. Furthermore, let $\Gamma$ be the set of all signatures where the number $k$ of players is taken over all values, that is,

$$
\Gamma=\bigcup_{k=2}^{\infty} \Gamma_{k} .
$$

Define sets $W$ and $L$ as follows:

$$
\begin{gathered}
W=\{\gamma \in \Gamma \mid \text { there is a key set protocol working for } \gamma\} \text {; and } \\
L=\{\gamma \in \Gamma \mid \text { there is no key set protocol working for } \gamma\} .
\end{gathered}
$$

Thus $\{W, L\}$ is a partition of set $\Gamma$. For $k=2$, i.e. $\gamma \in \Gamma_{2}$, Fischer and Wright give a simple necessary and sufficient condition for $\gamma \in W[3]$. For $k \geq 3$, the authors give a simple necessary and sufficient condition for $\gamma \in W[10$. (These necessary and sufficient conditions will be described in Section 2.5.)

One wishes to design a key set protocol which works for all signatures $\gamma \in W$, that is, always forms a tree as the key exchange graph for all deals $\mathcal{C}$ having any signature $\gamma \in W$. Such a protocol is said to be optimal for the class of key set protocols 36. There exists an optimal key set protocol indeed: the "SFP protocol" given by Fischer and Wright is an example of an optimal key set protocol 36]. However, neither an optimal key set protocol other than the SFP protocol nor a characterization of optimal key set protocols has been known so far.

In this paper, using the condition for $\gamma \in W$ in 10, we give a complete characterization of optimal key set protocols, that is, we give a necessary and sufficient condition for a key set protocol to be optimal. Using the characterization, we can design many optimal key set protocols. Thus we show that not only the SFP protocol but also many others are optimal. Using these optimal protocols, one can produce trees of various shapes as a key exchange graph; some of them would be appropriate for efficient broadcast of a secret message. For example, one can produce a tree of a small radius, as we will show later in Section 4 


\section{Preliminaries}

In this section we explain the "key set protocol" formalized by Fischer and Wright, and present some of the known results on this protocol [2 3,6 10].

\subsection{Key Set Protocol}

We first define some terms. A key set $K=\{x, y\}$ consists of two cards $x$ and $y$, one in $C_{i}$, the other in $C_{j}$ with $i \neq j$, say $x \in C_{i}$ and $y \in C_{j}$. We say that a key set $K=\{x, y\}$ is opaque if $1 \leq i, j \leq k$ and Eve cannot determine whether $x \in C_{i}$ or $x \in C_{j}$ with probability greater than $1 / 2$. Note that both players $P_{i}$ and $P_{j}$ know that $x \in C_{i}$ and $y \in C_{j}$. If $K$ is an opaque key set, then $P_{i}$ and $P_{j}$ can share a one-bit secret key $r_{i j} \in\{0,1\}$, using the following rule agreed on before starting a protocol: $r_{i j}=0$ if $x>y ; r_{i j}=1$, otherwise. Since Eve cannot determine whether $r_{i j}=0$ or $r_{i j}=1$ with probability greater than $1 / 2$, the secret key $r_{i j}$ is information-theoretically secure. We say that a card $x$ is discarded if all the players agree that $x$ has been removed from someone's hand, that is, $x \notin\left(\bigcup_{i=1}^{k} C_{i}\right) \cup C_{e}$. We say that a player $P_{i}$ drops out of the protocol if she no longer participates in the protocol. We denote by $V$ the set of indices $i$ of all the players $P_{i}$ remaining in the protocol. Note that $V=\{1,2, \cdots, k\}$ before starting a protocol.

The "key set protocol" has four steps as follows.

1. Choose a player $P_{s}, s \in V$, as a proposer by a certain procedure.

2. The proposer $P_{s}$ determines in mind two cards $x, y$. The cards are randomly picked so that $x$ is in her hand and $y$ is not in her hand, i.e. $x \in C_{s}$ and $y \in\left(\bigcup_{i \in V-\{s\}} C_{i}\right) \cup C_{e}$. Then $P_{s}$ proposes $K=\{x, y\}$ as a key set to all the players. (The key set is proposed just as a set. Actually it is sorted in some order, for example in ascending order, so Eve learns nothing about which card belongs to $C_{s}$ unless Eve holds $y$.)

3. If there exists a player $P_{t}$ holding $y$, then $P_{t}$ accepts $K$. Since $K$ is an opaque key set, $P_{s}$ and $P_{t}$ can share a one-bit secret key $r_{s t}$ that is informationtheoretically secure from Eve. (In this case an edge $(s, t)$ is added to the key exchange graph.) Both cards $x$ and $y$ are discarded. Let $P_{i}$ be either $P_{s}$ or $P_{t}$ that holds the smaller hand; if $P_{s}$ and $P_{t}$ hold hands of the same size, let $P_{i}$ be the proposer $P_{s} . P_{i}$ discards all her cards and drops out of the protocol. Set $V:=V-\{i\}$. Return to step 1 .

4. If there exists no player holding $y$, that is, Eve holds $y$, then both cards $x$ and $y$ are discarded. Return to step 1. (In this case no new edge is added to the key exchange graph.)

These steps 1-4 are repeated until either exactly one player remains in the protocol or there are not enough cards left to complete step 2 even if two or more players remain. In the first case the key exchange graph becomes a tree. 
In the second case the key exchange graph does not become a connected graph and hence does not become a tree.

Considering various procedures for choosing the proposer $P_{s}$ in step 1 , we obtain the class of key set protocols, where all the procedures are functions $\Gamma_{k} \rightarrow$ $V$.

\subsection{Malicious Adversary}

If a key set protocol works for a signature $\gamma$, then the key exchange graph must become a tree for any deal $\mathcal{C}$ having the signature $\gamma$. Hence, whoever has the card $y$ contained in the proposed key set $K=\{x, y\}$, the key exchange graph should become a tree. The malicious adversary determines who holds the card $y$. We use a function $\mathcal{A}: \Gamma_{k} \times V \rightarrow V \cup\{e\}$ to represent a malicious adversary, where $e$ is Eve's index. The inputs to the function $\mathcal{A}(\gamma, s)$ are the current signature $\gamma \in \Gamma_{k}$ and the index $s \in V$ of a proposer $P_{s}$ chosen by the protocol. Its output is either the index $t$ of a player $P_{t}$ remaining in the protocol or the index $e$ of Eve; $\mathcal{A}(\gamma, s)=t \neq e$ means that player $P_{t}$ holds card $y$; and $\mathcal{A}(\gamma, s)=e$ means that Eve holds card $y$.

From now on, we denote by $\gamma=\left(c_{1}, c_{2}, \cdots, c_{k} ; c_{e}\right)$ the current signature, and denote by $\gamma_{(s, \mathcal{A})}^{\prime}=\left(c_{1}^{\prime}, c_{2}^{\prime}, \cdots, c_{k^{\prime}}^{\prime} ; c_{e}^{\prime}\right)$ the resulting signature after executing steps 1-4 under the assumption that $P_{s}$ proposes a key set $K=\{x, y\}$ and $y \in C_{\mathcal{A}(\gamma, s)}$. We sometimes write $\gamma^{\prime}$ instead of $\gamma_{(s, \mathcal{A})}^{\prime}$ if it is clear from context.

Consider a signature $\gamma=(8,7,6,4,4,4,3,2,1 ; 3)$ as an example. Then, as illustrated in Fig. 1(a), the size of the hand of each player or Eve can be represented by white rectangles. For example, if the malicious adversary $\mathcal{A}$ satisfies $\mathcal{A}(\gamma, 2)=\mathcal{A}(\gamma, 3)=1$, then $\gamma_{(2, \mathcal{A})}^{\prime}=(7,6,4,4,4,3,2,1 ; 3)$ as in Fig. 1 $(\mathrm{b})$, and $\gamma_{(3, \mathcal{A})}^{\prime}=(7,7,4,4,4,3,2,1 ; 3)$ as in Fig. 1(c). In Figs. 1(b) and (c), the shaded rectangles correspond to the discarded cards.

If an optimal key set protocol chooses a proposer $P_{s}$ for $\gamma \in W$, then $\gamma_{(s, \mathcal{A})}^{\prime} \in$ $W$ for any malicious adversary $\mathcal{A}$; for convenience sake any signature $\gamma=\left(c_{1} ; c_{e}\right)$ with $k=1$ is assumed to be in $W$.

It follows from the definition of a key set protocol that if two players $P_{i}$ and $P_{j}$ hold hands of the same size, that is, $c_{i}=c_{j}$, then

$$
\forall \mathcal{A} \quad \gamma_{(i, \mathcal{A})}^{\prime} \in W \Longleftrightarrow \forall \mathcal{A} \quad \gamma_{(j, \mathcal{A})}^{\prime} \in W
$$

Hence, if there exist two or more players $P_{i}$ with $c_{i}=c_{s}$ (including the proposer $P_{s}$ ), then one may assume without loss of generality that $P_{s}$ has the largest index among all these players. We call it Assumption 1 for convenience sake. Similarly, if $\mathcal{A}(\gamma, s)=t \neq e$ and there exist two or more players $P_{i}$ with $c_{i}=c_{t}$ and $i \neq s$ (including $P_{t}$ ), then one may assume without loss of generality that $P_{t}$ has the largest index among all these players. We call it Assumption 2 for convenience sake. Under the two assumptions above, $\gamma_{(s, \mathcal{A})}^{\prime}=\left(c_{1}^{\prime}, c_{2}^{\prime}, \cdots, c_{k^{\prime}}^{\prime} ; c_{e}^{\prime}\right)$ satisfies $c_{1}^{\prime} \geq c_{2}^{\prime} \geq \cdots \geq c_{k^{\prime}}^{\prime}$ since $\gamma$ satisfies $c_{1} \geq c_{2} \geq \cdots \geq c_{k}$. 


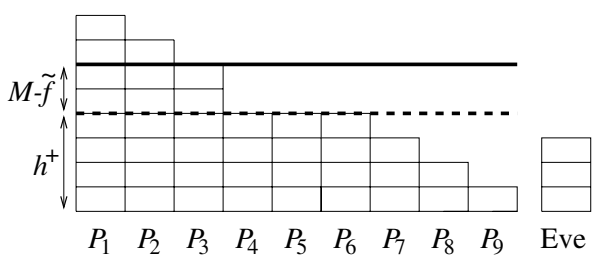

(a)

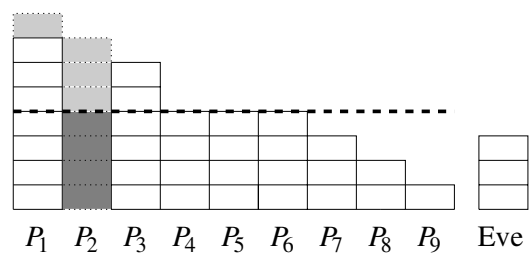

(b)

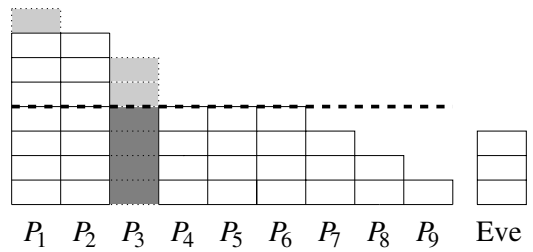

(c)

Fig. 1. The alteration of a signature.

\subsection{Feasible Players}

Fischer and Wright define a "feasible" player for a proposer as follows [36]. Let $k \geq 3$. If $c_{e} \geq 1, P_{i}$ with $c_{i}=1$ were chosen as a proposer, and $\mathcal{A}(\gamma, i)=e$, then $P_{i}$ 's hand would become empty although she remains in the protocol, and hence the key exchange graph would not become a tree. On the other hand, if $c_{e}=0$, then $\mathcal{A}(\gamma, i) \neq e$ and hence the protocol appears to be able to choose $P_{i}$ with $c_{i}=1$ as a proposer; however, if $\mathcal{A}(\gamma, i)=j$ and $c_{j}=1$, then $P_{j}$ 's hand would become empty and hence the key exchange graph would not become a tree. Thus the protocol can choose $P_{i}$ with $c_{i}=1$ as a proposer only if $c_{e}=0$ and $c_{j} \geq 2$ for every $j$ such that $1 \leq j \leq k$ and $j \neq i$, that is, only if $c_{e}=0$, $i=k$ and $c_{k-1} \geq 2$. Remember that $c_{1} \geq c_{2} \geq \cdots \geq c_{k}$ is assumed. Hence, we say that a player $P_{i}$ is feasible if the following condition (1) or (2) holds.

(1) $c_{i} \geq 2$.

(2) $c_{e}=0, c_{i}=1$ with $i=k$, and $c_{k-1} \geq 2$.

Thus, if the hands of all the players remaining in a protocol are not empty, i.e. $c_{k} \geq 1$, and the proposer $P_{s}$ is feasible, then the hands of all the players remaining in the protocol will not be empty at the beginning of the succeeding execution of steps $1-4$, i.e. $c_{k^{\prime}}^{\prime} \geq 1$. Note that there will not always exist a feasible player at the beginning of the succeeding execution of steps 1-4 even if the proposer $P_{s}$ is feasible.

We define a mapping $f$ from $\Gamma_{k}$ to $\{0,1,2, \cdots, k\}$, as follows: $f(\gamma)=i$ if $P_{i}$ is the feasible player with the smallest hand (ties are broken by selecting the player having the largest index); and $f(\gamma)=0$ if there is no feasible player. For example, if $\gamma=(4,3,2,2,1,1 ; 3)$, then $f(\gamma)=4$. If $\gamma=(4,4,3,3,1 ; 0)$, then $f(\gamma)=k=5$ because $c_{e}=0, c_{k}=1$ and $c_{k-1} \geq 2$. If $\gamma=(1,1,1 ; 2)$, then 
$f(\gamma)=0$ because there is no feasible player. Hereafter we often denote $f(\gamma)$ simply by $f$ and $f\left(\gamma^{\prime}\right)$ by $f^{\prime}$.

The following Lemma 1 immediately holds [3:10].

Lemma 1 ([3,10]) The following (a)-(d) hold.

(a) If $\gamma \in W$, then $c_{k} \geq 1$ [3].

(b) If $k \geq 3$ and $\gamma \in W$, then $f \geq 1$ [3].

(c) If $c_{k} \geq 1$, then $c_{i}=1$ for every $i$ such that $f+1 \leq i \leq k$ 10].

(d) If $f \geq 1$ and $c_{f}=1$, then $f=k, c_{k}=1, c_{k-1} \geq 2, c_{e}=0$, and $\gamma \in W$ [3].

\subsection{SFP Protocol}

Fischer and Wright give the SFP (smallest feasible player) protocol as a key set protocol 36]. The SFP protocol always chooses the feasible player with the smallest hand as a proposer, that is, chooses the proposer $P_{s}$ as follows:

$$
s=\left\{\begin{array}{l}
f(\gamma) \text { if } 1 \leq f(\gamma) \leq k \\
1 \quad \text { if } f(\gamma)=0
\end{array}\right.
$$

Fischer and Wright show that the SFP protocol is optimal 3,6].

Theorem $2([\underline{3}, \underline{\mathbf{6}}])$ The SFP protocol is optimal.

Not only the SFP protocol but also many other key set protocols are optimal. This paper provides a complete characterization of optimal key set protocols.

\subsection{Necessary and Sufficient Condition for $\gamma \in W$}

For $k=2$, the following Theorem 3 provides a necessary and sufficient condition for $\gamma \in W[3]$.

Theorem 3 ([3]) Let $k=2$. Then $\gamma \in W$ if and only if $c_{2} \geq 1$ and $c_{1}+c_{2} \geq$ $c_{e}+2$.

For $k=3$, the following Theorem 4 provides a necessary and sufficient condition for $\gamma \in W[10$.

Theorem 4 ([10]) Let $k=3$. Then $\gamma \in W$ if and only if $c_{3} \geq 1$ and $c_{1}+c_{3} \geq$ $c_{e}+3$.

For $k \geq 4$, the following Theorem 5 provides a necessary and sufficient condition for $\gamma \in W[10]$. Hereafter let $B=\left\{i \in V \mid c_{i}=2\right\}$, and let $b=\lfloor|B| / 2\rfloor$.

Theorem 5 ([10]) Let $k \geq 4, c_{k} \geq 1$ and $f \geq 1$. Then $\gamma \in W$ if and only if

$$
\sum_{i=1}^{k} \max \left\{c_{i}-h^{+}, 0\right\} \geq \tilde{f},
$$


where

$$
\begin{gathered}
\bar{f}=f-\delta, \\
\tilde{f}=\bar{f}-2 \epsilon, \\
h=c_{e}-c_{k}+k-\bar{f}, \\
h^{+}=h+\epsilon, \\
\delta=\left\{\begin{array}{l}
0 \text { if } f=1 ; \\
1 \text { if } 2 \leq f \leq k-1 ; \\
2 \text { if } f=k \text { and } c_{k-1} \geq c_{k}+1 ; \text { and } \\
3 \text { if } f=k \text { and } c_{k-1}=c_{k},
\end{array}\right.
\end{gathered}
$$

and

$$
\epsilon= \begin{cases}\max \left\{\min \left\{c_{2}-h, b\right\}, 0\right\} & \text { if } 5 \leq f \leq k-1 \\ \max \left\{\min \left\{c_{2}-h, b-1\right\}, 0\right\} & \text { if } 5 \leq f=k \text { and } c_{e} \geq 1 ; \text { and } \\ 0 & \text { otherwise. }\end{cases}
$$

For example, one can observe a signature $\gamma=(8,7,6,4,4,4,3,2,1 ; 3)$ (see Fig. 1(a)) satisfies Eq. (1) in Theorem 5 as follows. The signature $\gamma$ satisfies $k=9$ and $f=8$. Thus by Eq. (6) $\delta=1$. Since $B=\{8\}, b=0$ and hence by Eq. (7) $\epsilon=0$. Thus, by Eqs. (2) and (3) $\tilde{f}=\bar{f}=8-1=7$, and by Eqs. (4) and (5) $h^{+}=h=3-1+9-7=4$. Therefore,

$$
\sum_{i=1}^{k} \max \left\{c_{i}-h^{+}, 0\right\}=4+3+2=9>7=\tilde{f}
$$

and hence the signature $\gamma$ satisfies Eq. (11). (Note that $\sum_{i=1}^{k} \max \left\{c_{i}-h^{+}, 0\right\}$ is equal to the number of rectangles above the dotted line in Fig. 11(a).) Thus $\gamma \in W$.

Eq. (1) looks in appearance to be similar to the condition for a given degree sequence to be "graphical" 1,7,811.

Since $c_{1} \geq c_{2} \geq \cdots \geq c_{k}$ is assumed, Eq. (1) is equivalent to

$$
\sum_{i=1}^{\tilde{f}} \max \left\{c_{i}-h^{+}, 0\right\} \geq \tilde{f}
$$

where the summation is taken over all $i, 1 \leq i \leq \tilde{f}$, although the summation in Eq. (11) is taken over all $i, 1 \leq i \leq k$ [10].

We define $\delta^{\prime}, \epsilon^{\prime}, b^{\prime}, \bar{f}^{\prime}, \widetilde{f}^{\prime}, h^{\prime}, h^{+^{\prime}}$ and $B^{\prime}$ for $\gamma^{\prime}$ as we did for $\gamma$.

\section{Main Results}

In this section we give a complete characterization of optimal key set protocols. We first define some terms in Section [3.1, and then give the characterization in Section 3.2 . 


\subsection{Definition of Selectable Players}

In this subsection we define a "selectable" player that can be chosen as a proposer by an optimal key set protocol. We will give a complete characterization of "selectable" players in the succeeding subsection. The characterization immediately provides a complete characterization of optimal key set protocols.

The SFP protocol, which always chooses the feasible player $P_{f}$ with the smallest hand, is optimal. However, a key set protocol which chooses an arbitrary feasible player is not necessarily optimal. We define a "selectable" player as follows.

Definition 6 We say that a player $P_{i}$ is selectable for $\gamma$ if $\gamma_{(i, \mathcal{A})}^{\prime} \in W$ for any malicious adversary $\mathcal{A}$.

When $\gamma \in W$, the proposer chosen by an optimal key set protocol is a selectable player, of course. Since the SFP protocol is optimal, $P_{f}$ is a selectable player if $\gamma \in W$.

Definition [6 implies that $\gamma \in W$ if and only if there exists at least one selectable player. In other words, $\gamma \in L$ if and only if there exists no selectable player.

Furthermore, a key set protocol is optimal if and only if the protocol always chooses a selectable player as a proposer whenever such a player exists. Thus, in the remainder of the paper, we characterize the set of all selectable players.

\subsection{Characterization of Selectable Players}

In this subsection we give a necessary and sufficient condition for a player to be selectable.

If $\gamma \in L$, then there is no selectable player. Therefore it suffices to obtain a necessary and sufficient condition for a player to be selectable only if $\gamma \in W$.

We first characterize the selectable players for $k=2$ as in the following Theorem 7.

Theorem 7 Let $k=2$ and $\gamma \in W$. Then a player $P_{i}$ is selectable if and only if $c_{i} \geq 2$ or $c_{e}=0$.

Proof. Let $k=2$ and $\gamma \in W$. By Lemma 1 (a) $c_{2} \geq 1$.

We first prove the necessity. Suppose for a contradiction that $c_{i}=1$ and $c_{e} \geq$ 1 although $P_{i}$ is selectable. Then one may assume that $i=2$ by Assumption 1 for convenience sake when $P_{i}$ is chosen as a proposer. Since $\gamma_{(2, \mathcal{A})}^{\prime}=\left(c_{1}, 0 ; c_{e}-1\right)$ for an adversary $\mathcal{A}$ such that $\mathcal{A}(\gamma, 2)=e$, we have $\gamma_{(2, \mathcal{A})}^{\prime} \in L$ by Lemma 1(a). Thus $P_{2}$, i.e. $P_{i}$, is not selectable, a contradiction.

We next prove the sufficiency. Assume that $c_{i} \geq 2$ or $c_{e}=0$. Then it suffices to show that $\gamma_{(i, \mathcal{A})}^{\prime} \in W$ for any adversary $\mathcal{A}$. There are the following two cases to consider.

Case 1: $\mathcal{A}(\gamma, i) \neq e$.

In this case $\gamma^{\prime}$ satisfies $k^{\prime}=1$ and hence $\gamma^{\prime} \in W$. 
Case 2: $\mathcal{A}(\gamma, i)=e$.

In this case $c_{e} \geq 1$, and hence $c_{i} \geq 2$ because we assumed that $c_{i} \geq 2$ or $c_{e}=0$. If $i=1$ and $c_{1} \geq c_{2}+1$, then $\gamma^{\prime}=\left(c_{1}-1, c_{2} ; c_{e}-1\right)$; otherwise, $\gamma^{\prime}=\left(c_{1}, c_{2}-1 ; c_{e}-1\right)$. Thus, in either case, $c_{1}^{\prime}+c_{2}^{\prime}=\left(c_{1}+c_{2}\right)-1$ and $c_{e}^{\prime}=c_{e}-1$. On the other hand, since $\gamma \in W$, by Theorem $3 c_{1}+c_{2} \geq c_{e}+2$. Therefore $c_{1}^{\prime}+c_{2}^{\prime} \geq\left(c_{e}+2\right)-1=c_{e}+1=c_{e}^{\prime}+2$. Furthermore, since $c_{i} \geq 2$, $c_{2}^{\prime} \geq 1$. Thus, by Theorem $3 \gamma^{\prime} \in W$.

We next characterize the selectable players for $k=3$. It has been known that, if $c_{k} \geq 1$ and $c_{1}+c_{k} \geq c_{e}+k$, then any key set protocol choosing an arbitrary feasible player as a proposer works for $\gamma$ [36]; thus the following Lemma 8 immediately holds.

Lemma 8 Let $c_{k} \geq 1$ and $c_{1}+c_{k} \geq c_{e}+k$. Then every player $P_{i}$ such that $1 \leq i \leq f$ is selectable.

Furthermore, it is obvious that any non-feasible player is not selectable when $k \geq 3$; thus we have the following Lemma 9 .

Lemma 9 Let $k \geq 3$. If a player $P_{i}$ is selectable, then $1 \leq i \leq f$.

By using Theorem 4 Lemmas 8 and 9 one can easily prove that the selectable players for $k=3$ are characterized as in the following Theorem 10 .

Theorem 10 Let $k=3$ and $\gamma \in W$. Then a player $P_{i}$ is selectable if and only if $1 \leq i \leq f$.

Proof. Let $k=3$ and $\gamma \in W$. Then by Theorem $4 c_{3} \geq 1$ and $c_{1}+c_{3} \geq$ $c_{e}+3$. Thus Lemma 8 implies the sufficiency. Furthermore Lemma 9 implies the necessity.

We finally characterize the selectable players for $k \geq 4$. Before giving the characterization, we first give some definitions.

In a key set protocol, for every $i, j \in V$ such that $i \neq j$ and $c_{i}=c_{j}$,

$$
P_{i} \text { is selectable } \Longleftrightarrow P_{j} \text { is selectable. }
$$

Thus, if there exist two or more players holding hands of the same size, then it suffices to determine whether an arbitrary player among such players is selectable or not. For example, if $\gamma=(8,7,6,4,4,4,3,2,1 ; 3)$, then one can choose $P_{6}$ as a "representative" player among the three players $P_{4}, P_{5}$ and $P_{6}$ who have hands of size 4. As in this example, we choose the player with the largest index among all the players holding hands of the same size as a "representative" player, and determine whether the chosen "representative" player is selectable or not. Let $V_{r}$ be the set of indices of all the "representative" players. That is,

$$
V_{r}=\{i \in V \mid i=\max X \text { and } X \in V / R\} \text {, }
$$


where $V / R$ is the quotient set of $V$ under the equivalence relation $R=\{(i, j) \in$ $\left.V \times V \mid c_{i}=c_{j}\right\}$. For example, $V_{r}=\{1,2,3,6,7,8,9\}$ for the above signature $\gamma$. It suffices to give a necessary and sufficient condition for a player $P_{i}, i \in V_{r}$, to be selectable. Of course, such a necessary and sufficient condition immediately yields a complete characterization of all selectable players (whose indices are not necessarily in $V_{r}$ ).

Let $P_{f_{m}}$ be the player who holds the hand of the same size as $P_{f}$ and has the smallest index, that is,

$$
f_{m}=\min \left\{i \in V \mid c_{i}=c_{f}\right\} .
$$

From now on we define

$$
M=\sum_{j=1}^{k} \max \left\{c_{j}-h^{+}, 0\right\} .
$$

Note that $M$ is the same as the left side of Eq. (1) in Theorem [5. We define $M^{\prime}$ for $\gamma^{\prime}$ as we did for $\gamma$.

Define $\bar{\epsilon}$ by the following Eq. (9), which is obtained by replacing $c_{2}$ with $c_{3}$ in Eq. (7):

$$
\bar{\epsilon}= \begin{cases}\max \left\{\min \left\{c_{3}-h, b\right\}, 0\right\} & \text { if } 5 \leq f \leq k-1 ; \\ \max \left\{\min \left\{c_{3}-h, b-1\right\}, 0\right\} & \text { if } 5 \leq f=k \text { and } c_{e} \geq 1 ; \text { and } \\ 0 & \text { otherwise. }\end{cases}
$$

Since $c_{3} \leq c_{2}$, Eqs. (7) and (9) imply

$$
0 \leq \bar{\epsilon} \leq \epsilon .
$$

Furthermore, define Conditions 1 and 2 as follows.

(Condition 1)

$5 \leq f=k$ and $c_{k-2}=c_{k-1}=c_{k}+1$.

(Condition 2)

$c_{f_{m}-2}=c_{f_{m}-1}=3,|B|$ is an odd number, and the following (i) or (ii) holds.

(i) $6 \leq f \leq k-1$ and $c_{2}-h \geq b+1$.

(ii) $6 \leq f=k, c_{e} \geq 1, b \geq 1$ and $c_{2}-h \geq b$.

Define $\lambda$ as follows:

$$
\lambda=\left\{\begin{array}{l}
2 \text { if Condition } 1 \text { holds; } \\
3 \text { if Condition } 2 \text { holds; and } \\
0 \text { otherwise. }
\end{array}\right.
$$

Finally, define $\widetilde{\epsilon}$ as follows:

$$
\widetilde{\epsilon}= \begin{cases}\max \left\{\min \left\{c_{2}-h-1, b-1\right\}, 0\right\} & \text { if } f \geq 8, c_{k}=1 \text { and } \lambda=2 ; \text { and } \\ 0 & \text { otherwise. }\end{cases}
$$

We are now ready to give a complete characterization of the selectable players for $k \geq 4$ as in the following Theorem[11. 
Theorem 11 Let $k \geq 4$ and $\gamma \in W$. Then a player $P_{i}$ such that $i \in V_{r}$ is selectable if and only if $1 \leq i \leq f$ and

$$
\left\{\begin{array}{lr}
c_{2}-h^{+} \leq M-\widetilde{f}-(\epsilon-\bar{\epsilon}) & \text { if } i \leq 2 ; \\
\sum_{j=1}^{\widetilde{f}-\lambda-2 \tilde{\epsilon}} \max \left\{c_{j}-\left(h^{+}+\widetilde{\epsilon}+1\right), 0\right\} \geq \widetilde{f}-\lambda-2 \widetilde{\epsilon} & \\
c_{i}-h^{+} \leq M-\widetilde{f} & \text { if } i=f_{m}-1 \geq 4 \text { and } \lambda \neq 0 ; \text { and }
\end{array}\right.
$$

If (i) $i \leq 2$ and $\epsilon-\bar{\epsilon}=0$, (ii) $i=3$, or (iii) $i \geq 4$ and $i \neq f_{m}-1$ or $\lambda=0$, then Eq. (13) in Theorem 11 becomes

$$
\left\{\begin{array}{l}
c_{2}-h^{+} \leq M-\widetilde{f} \text { if } i \leq 2 ; \text { and } \\
c_{i}-h^{+} \leq M-\widetilde{f} \text { if } i \geq 3
\end{array}\right.
$$

Note that the most of signatures satisfy $\epsilon-\bar{\epsilon}=\lambda=0$ and that very few signatures satisfy $\epsilon-\bar{\epsilon} \geq 1$ or $\lambda \neq 0$.

Consider the signature $\gamma=(8,7,6,4,4,4,3,2,1 ; 3)$ as an example again (see Fig. 1(a)). The signature $\gamma$ satisfies $\epsilon=0$ as mentioned in Section 2.5, and hence by Eq. (10) $\epsilon-\bar{\epsilon}=0$. The signature $\gamma$ does not satisfy Condition 1 . Furthermore, since $f=f_{m}=8$, we have $c_{f_{m}-2}=4 \neq 3$ and hence Condition 2 does not hold. Therefore, by Eq. (11) $\lambda=0$. In addition, since the signature $\gamma$ satisfies $f=8$, $M=9, \tilde{f}=7$ and $h^{+}=4$ as mentioned in Section 2.5 we have $M-\widetilde{f}=2$. Therefore, Eq. (13) in Theorem 11 i.e. Eq. (14), implies that all the selectable players are the six players $P_{3}, P_{4}, P_{5}, P_{6}, P_{7}$ and $P_{8}$. These six players are the feasible players holding the hands whose sizes do not exceed the solid line in Fig. 1(a).

We now intuitively explain the correctness of Theorem 11. For simplicity, let $\epsilon-\bar{\epsilon}=\lambda=0$, and consider a player $P_{i}$ such that $i \geq 2$. Theorem 5 implies that a necessary and sufficient condition for $\gamma \in W$ is that $M \geq \widetilde{f}$, i.e. there are $\widetilde{f}$ or more rectangles above the dotted line in Fig. 1(a). Thus, a signature $\gamma \in W$ has $M-\widetilde{f}$ "spare" rectangles. That is, even if one removes at most $M-\widetilde{f}$ rectangles above the dotted line, $\gamma$ still remains in $W$, but if one removed $(M-\widetilde{f})+1$ or more rectangles above the dotted line, then $\gamma$ would be in $L$. Further, in order for a player $P_{i}$ to be selectable, there must exist at least $\widetilde{f}^{\prime}$ rectangles above the dotted line in the figure of $\gamma_{(i, \mathcal{A})}^{\prime}$ (e.g. Fig. 1)(b) or (c)) for any malicious adversary $\mathcal{A}$. For some adversary $\mathcal{A}$, the number of the rectangles above the dotted line decreases by $1+\left(c_{i}-h^{+}\right)$when the proposer is $P_{i}$, as one can immediately observe from Fig. 1)(b) or (c). Note that these $1+\left(c_{i}-h^{+}\right)$ rectangles are lightly shaded in Figs.1(b) and (c). Furthermore, since the number of the feasible players decreases by exactly one, we have $\tilde{f}^{\prime}=\tilde{f}-1$. Hence, if $c_{i}-h^{+}$were greater than the number $M-\tilde{f}$ of the "spare" rectangles, then $M^{\prime}=M-\left\{1+\left(c_{i}-h^{+}\right)\right\}<\widetilde{f}-1=\widetilde{f}^{\prime}$ and hence $\gamma^{\prime}$ would be in $L$. Therefore, a player $P_{i}$ such that $c_{i}-h^{+}>M-\widetilde{f}$ is not selectable. On the other hand, if $c_{i}-h^{+} \leq M-\widetilde{f}$, then $\gamma^{\prime}$ will still remain in $W$, and hence a player $P_{i}$ such that $c_{i}-h^{+} \leq M-\widetilde{f}$ is selectable. This is the intuitive reason why Theorem [1] holds. 
Due to the page limitation, we cannot include a proof of Theorem 11 in this extended abstract; see [9].

\section{Conclusion}

A key set protocol is determined by giving a procedure for choosing a proposer. In this paper, we defined a player to be selectable if the player can be chosen as a proposer by an optimal key set protocol, and gave a complete characterization of such selectable players in Theorem 11. Thus we succeeded in characterizing the set of all optimal key set protocols.

Using Theorem 11, one can efficiently find all selectable players in time $O(k)$. Let $P_{j}$ be the selectable player having the smallest index $j$. Then one may intuitively expect that all players $P_{i}$ such that $j \leq i \leq f$ are selectable. However, it is surprisingly not the case. Theorem 11 implies that all the players such that $j \leq i \leq f$ and $c_{i} \neq c_{f_{m}-1}$ are selectable but $P_{f_{m}-1}$ may or may not be selectable. Consider a signature $\gamma=(5,5,5,4,4,3,3,2,1 ; 2)$ as an example. Then $\gamma$ satisfies $f=8, f_{m}-1=7, \lambda=3, h^{+}=3, M=8, \epsilon=\bar{\epsilon}=\widetilde{\epsilon}=0$ and $\tilde{f}=7$. Thus Eq. (13) in Theorem 11 becomes

$$
\left\{\begin{array}{lr}
c_{2}-3 \leq 1 & \text { if } i \leq 2 ; \\
\sum_{\ell=1}^{4} \max \left\{c_{\ell}-4,0\right\} \geq 4 & \text { if } i=7 ; \text { and } \\
c_{i}-3 \leq 1 & \text { otherwise. }
\end{array}\right.
$$

Therefore, $P_{7}$ is not selectable, and all the selectable players are the three players $P_{4}, P_{5}$ and $P_{8}$. As in this example, the indices of selectable players are not necessarily consecutive numbers.

Using the characterization of selectable players, one can design many optimal key set protocols. Assume that $c_{1}=c_{2}=\cdots=c_{k}$ and $\gamma \in W$. Then in most cases the SFP protocol forms a spanning path of length $k$, i.e. a tree of radius $\lfloor k / 2\rfloor$, as the key exchange graph. On the other hand, using various optimal key set protocols, one can produce trees of various shapes as a key exchange graph, some of which would be appropriate for efficient broadcast of a secret message. For example, consider an optimal key set protocol which always chooses, as a proposer, the selectable player holding the largest hand; such a protocol forms a tree of a smaller radius than $\lfloor k / 2\rfloor$. Furthermore, we can choose the selectable player having the largest degree as a proposer and modify step 3 of the key set protocol in a way that either $P_{s}$ or $P_{t}$ who has the smaller degree drops out of the protocol whenever the resulting signature remains in $W$; such a protocol forms a tree of much smaller radius, especially when $c_{1}=c_{2}=\cdots=c_{k}$ is large. We have verified these facts by extensive computer simulation.

This paper addresses only the key set protocol, which establishes a one-bit secret key. On the other hand, the "transformation protocol" given by Fischer and Wright [4] establishes an $n$-bit secret key. For a signature $\gamma=(3,2 ; 4) \in L$, any key set protocol does not work for $\gamma$, but the transformation protocol always establishes a one-bit secret key for $\gamma$. However, for a signature $\gamma=(4,4,4,4 ; 4) \in$ 
$W$, any optimal key set protocol works for $\gamma$, but the transformation protocol cannot establish a one-bit secret key for $\gamma$. Thus a protocol entirely superior to the key set protocol has not been known.

\section{References}

1. T. Asano, "An $O(n \log \log n)$ time algorithm for constructing a graph of maximum connectivity with prescribed degrees," J. Comput. and Syst. Sci., vol. 51, pp. 503-510, 1995.

2. M. J. Fischer, M. S. Paterson, and C. Rackoff, "Secret bit transmission using a random deal of cards," DIMACS Series in Discrete Mathematics and Theoretical Computer Science, AMS, vol. 2, pp. 173-181, 1991.

3. M. J. Fischer and R. N. Wright, "An application of game-theoretic techniques to cryptography," DIMACS Series in Discrete Mathematics and Theoretical Computer Science, AMS, vol. 13, pp. 99-118, 1993.

4. M. J. Fischer and R. N. Wright, "An efficient protocol for unconditionally secure secret key exchange," Proc. of the 4th Annual Symposium on Discrete Algorithms, pp. 475-483, 1993.

5. M. J. Fischer and R. N. Wright, "Bounds on secret key exchange using a random deal of cards," J. Cryptology, vol. 9, pp. 71-99, 1996.

6. M. J. Fischer and R. N. Wright, "Multiparty secret key exchange using a random deal of cards," Proc. Crypto '91, Lecture Notes in Computer Science, SpringerVerlag, vol. 576, pp. 141-155, 1992.

7. S. L. Hakimi, "On realizability of a set of integers as degrees of the vertices of a linear graph. I," J. SIAM Appl. Math., vol. 10, no. 3, pp. 496-506, 1962.

8. F. Harary, "Graph Theory," Addison-Wesley, Reading, Mass., 1969.

9. T. Mizuki, "Sharing unconditionally secure secret keys," Ph.D. Thesis, Tohoku University, Sendai, 2000.

10. T. Mizuki, H. Shizuya, and T. Nishizeki, "Dealing necessary and sufficient numbers of cards for sharing a one-bit secret key," Proc. Eurocrypt '99, Lecture Notes in Computer Science, Springer-Verlag, vol. 1592, pp. 389-401, 1999.

11. E. F. Schmeichel and S. L. Hakimi, "On planar graphical degree sequences," SIAM J. Appl. Math., vol. 32, no. 3, pp. 598-609, 1977. 